\title{
Boundary scattering in the principal chiral model
}

\section{Niall MacKay* and Ben Short}

Department of Mathematics, University of York, York YO10 5DD, UK

E-mail: nmis@york.ac.uk, bjs108@york.ac.uki

ABSTRACT: In recent work on the $(G \times G$-invariant) principal chiral model with boundary, we found that both classically integrable boundary conditions and quantum boundary $S$ matrices were classified by the symmetric spaces $G / H$. The connection is explained by the presence of a 'twisted Yangian' algebra of non-local charges.

\section{Introduction}

In a recent paper $\left[\begin{array}{l}1 \\ 1\end{array}\right.$, we investigated the boundary principal chiral model, defined by

$$
\mathcal{L}=\frac{1}{2} \operatorname{Tr}\left(\partial_{\mu} g^{-1} \partial^{\mu} g\right)
$$

where the field $g(x, t)$ takes values in a compact classical Lie group $G$, defined on Minkowski space with $-\infty<x \leq 0$.

We found connections with symmetric spaces $G / H$ in two ways. First, we discovered mixed boundary conditions which allowed conservation and commutation of the local charges essential to integrability. In these, the field $g$ was restricted at $x=0$ to lie either in $H$ or in the Cartan immersion of $G / H$ (an embedding of $G / H$ as a submanifold of $G)$ or in a translate of one of these. We describe this briefly in section two. Next, we investigated rational solutions of the boundary Yang-Baxter or 'reflection' equation (which would be needed for the construction of boundary $S$-matrices), and found that those of the simplest form were also in correspondence with the symmetric spaces, there being solutions parametrized by the Cartan immersion of $G / H$. The beauty of this second correspondence in particular is easily swamped by the exhaustive (and exhausting) detail needed to prove it case-by-case, and in section three we instead give a flavour of how it works. The connection between the two is clarified by the discovery that a remnant of the bulk Yangian algebra of non-local charges survives on the half-line. This consists of the generators of the $H$ symmetry expected, along with a set of non-linear combinations of non-local charges, and together these form a twisted Yangian. We describe this in section four.

\footnotetext{
${ }^{*}$ Speaker.
} 


\section{Classical boundary conditions}

First, the classical boundary conditions (BCs). The boundary equation of motion is

$$
\operatorname{Tr}\left(g^{-1} \partial_{1} g \cdot g^{-1} \delta g\right)=0 \quad \text { at } \quad x=0,
$$

where the variation is over all $\delta g$ such that $g^{-1} \delta g \in \mathbf{g}$, the Lie algebra of $G$. This is clearly satisfied by the Neumann BC $\partial_{1} g=0$, or by the Dirichlet condition $\delta g=0$. But it is also satisfied by two types of mixed condition,

$$
\begin{array}{clrl}
\text { chiral } & g(0) & \in g_{L} H g_{R}^{-1} \\
\text { and non - chiral } & g(0) & \in g_{L} \frac{G}{H} g_{R}^{-1},
\end{array}
$$

where $G / H \cong\left\{\alpha(g) g^{-1} \mid g \in G\right\}$ is the Cartan immersion of $G / H$ in $G$, with $H$ the subgroup of $G$ invariant under an involutive (i.e. $\alpha^{2}=1$ ) automorphism $\alpha$, while $g_{L}, g_{R}$ are fixed elements of $G$. (Actually, one has to be a little careful; the Cartan immersion is a local diffeomorphism, but may be (finitely) neither 1-1 nor onto, as explained in [inj].)

This requirement that $g$ be restricted to lie in such a 'D-submanifold' is then supplemented by a Neumann condition on it. To see this, define the g-valued conserved currents which generate the $G \times G$ symmetry $g \mapsto U g V^{-1}$,

$$
j(x, t)_{\mu}^{L}=\partial_{\mu} g g^{-1}, \quad j(x, t)_{\mu}^{R}=-g^{-1} \partial_{\mu} g,
$$

and notice that the boundary equation of motion is equivalent to $\operatorname{Tr}\left(j_{0} j_{1}\right)=0$ at $x=0$ (using either $j^{L}$ or $j^{R}-$ it doesn't matter which because of cyclicity of trace). Our chiral BC ( $(\overline{2} . \overline{1})$ is equivalent to

$$
g_{L}^{-1} j_{0}^{L} g_{L}=\alpha\left(g_{L}^{-1} j_{0}^{L} g_{L}\right) \in \mathbf{h} \quad \text { and } \quad g_{R}^{-1} j_{1}^{L} g_{R}=\alpha\left(g_{R}^{-1} j_{1}^{L} g_{R}\right) \in \mathbf{h}
$$

at $x=0$, where $\mathbf{g}=\mathbf{h} \oplus \mathbf{k} ; \mathbf{h}$ generates $H$ and is the +1 eigenspace, while $\mathbf{k}$ is the -1 eigenspace. The boundary equation of motion then requires that the space components take values in $\mathbf{k}$. Together these give

$$
g_{L}^{-1} j_{+}^{L} g_{L}=\alpha\left(g_{L}^{-1} j_{-}^{L} g_{L}\right) \quad \text { at } x=0,
$$

and similarly for $j^{R}$. It is an easy exercise to show similarly that the non-chiral BC (2.2.2) implies $g_{L}^{-1} j_{0}^{L} g_{L}=\alpha\left(g_{R}^{-1} j_{0}^{R} g_{R}\right)$ and so requires

$$
g_{L}^{-1} j_{+}^{L} g_{L}=\alpha\left(g_{R}^{-1} j_{-}^{R} g_{R}\right) \quad \text { at } x=0 .
$$

The conserved, commuting local charges of the model are built up using certain special choices of invariant tensors [2] 2$]$. That they remain conserved and commuting on the half-line in the presence of these BCs follows from the simple behaviour of these tensors under $\alpha$. When $\alpha$ is an inner automorphism, the tensors are of course invariant under $\alpha$, but when $\alpha$ is outer they may not be so. In fact they always have eigenvalue \pm 1 under $\alpha$ [i] this is enough to ensure that one conserved charge may be constructed from each \pm spin 
pair of local charges - for example, energy but not momentum. This, and the consistency of the Poisson brackets with these BCs, is described in detail in [i]1]

The final point concerns the moduli space of parameters $\left(g_{L}, g_{R}\right)$ by which we may 'twist' our BCs. For the chiral BCs, it is clear that this will be $G / H \times G / H$, modulo some finite set. For each such BC we must construct a boundary $S$-matrix, and this should respect the remnant $g_{L} H g_{L}^{-1} \times g_{R} H g_{R}^{-1}$ of the $G \times G$ symmetry.

\section{Boundary $S$-matrices}

Assuming that these results survive quantization, we must now seek solutions of the reflection equation from which to construct boundary $S$-matrices. Recall the reflection equation

$$
S(\theta-\phi) . I \otimes K(\theta) . S(\theta+\phi) . I \otimes K(\phi)=I \otimes K(\phi) . S(\theta+\phi) . I \otimes K(\theta) . S(\theta-\phi),
$$

acting on $V \otimes V$ where $V$ is the defining (vector) representation (or possibly, for $S U(N)$ ), its conjugate) of a classical group $G$. (The corresponding calculations for any exceptional groups are a rather tougher proposition.) We begin with the known rational $S: V \otimes V \rightarrow$ $V \otimes V$, and must find $K: V \rightarrow V$. To do so we make the ansatz

$$
K(\theta)=K_{1}(\theta) \equiv \rho(\theta) E \quad \text { or } \quad K(\theta)=K_{2}(\theta) \equiv \frac{\tau(\theta)}{1-c \theta}(I+c \theta E),
$$

where $c$ and $E$ are constants to be determined, the latter an $N \times N$ matrix, and $\rho(\theta)$ and $\tau(\theta)$ are scalar prefactors, undetermined by the reflection equation but which are needed to satisfy the unitarity and crossing-unitarity conditions (which we do not detail here) required of boundary $S$-matrices. The second form $K_{2}$ is basically Cherednik's ansatz [4] Physically, these are $S$-matrices with at most two 'channels'; $K_{1}(\theta)$ will have no nontrivial pole structure, while with $K_{2}(\theta)$ there is the possibility of precisely one non-trivial boundary bound state.

On substituting these into the reflection, unitarity and crossing-unitarity conditions, we obtain various sets of conditions on $E$ (and $c$ ). Our result is that each set corresponds to a symmetric space $G / H$, and that in each case the space of $E$ which satisfies the conditions is isomorphic to (sometimes a finite multiple of) a translate of the Cartan immersion $G / H \hookrightarrow G$.

For a brief indication of how such results are arrived at, let us look at the example of $S U(N)$. There are two forms of the reflection equation to consider: that acting on $V \otimes V$, and that on $V \otimes \bar{V}$. For $V \otimes V$, one finds that $K_{2}$ solves all the conditions if

$$
E^{\dagger} E=I, \quad E=E^{\dagger} \quad \text { and } \quad c=-\frac{2 N}{i \pi \operatorname{Tr} E} .
$$

The only $K_{1}$-type solution is really the degeneration of this when $\operatorname{Tr} E=0$. For $V \otimes \bar{V}$, one finds that $K_{2}$ gives no solutions, but $K_{1}$ works with

$$
E^{\dagger} E=I, \quad \operatorname{det} E= \pm 1 \quad \text { and } \quad E= \pm E^{T}
$$

(the two choices of sign being independent). 
Now consider $\alpha: S U(N) \rightarrow S U(N), U \mapsto X U X$ where $X=\operatorname{diag}(+1 M$ times, -1 $N-M$ times). Then

$$
\frac{S U(N)}{S(U(M) \times U(N-M)} \cong\left\{X U X U^{-1} X \mid U \in S U(N)\right\}
$$

a translation by $X$ (and thus in $U(N)$ when $N-M$ is odd) of the Cartan immersion. It is easy to see that this is contained in the space of $E$ satisfying (2. $2 M-N=-4 / c$ ), although to prove that they are equivalent takes rather longer. (The $K_{1}$ solution corresponds to the case $2 M=N$.)

This covers the $V \otimes V$ solutions. Now for $V \otimes \bar{V}$ consider

$$
\frac{S U(N)}{S O(N)} \cong\left\{\bar{U} U^{-1} \mid U \in S U(N)\right\}=\left\{E \in S U(N) \mid E=E^{T}\right\}
$$

- so that here the set of $E$ satisfying ( $\left(\overline{3}-4^{i}\right)$ is a two-fold copy of the symmetric space. Note again that it is simple to check that the first set is contained in the second, rather harder to check the reverse. Finally, for $N$ even, the choice $E=-E^{T}$ corresponds to $S U(N) / S p(N)$.

We can now construct the boundary $S$-matrices for the principal chiral model in the form

$$
Y(\theta)\left(K_{L}(\theta) \otimes K_{R}(\theta)\right)
$$

where $K_{L, R}(\theta)$ are $L$ and $R$ copies of the same type of $K$ as found above, with their scalar prefactors constructed so as to give no poles on the physical strip $0 \leq \operatorname{Im} \theta \leq i \pi / 2$. The CDD factor $Y$ is then used to implement the pole structure we desire.

Let us note some facts about the $K_{2}$-type solution for the Grassmannian symmetric space (3.5. and indeed it is, since (after a relabelling of bases) $X \mapsto-X, E \mapsto-E$ and $c \mapsto-c$, leaving $I+c \theta E$ invariant; the scalar prefactors can then be constructed to respect this. Second, the boundary $S$-matrix is, like the classical BCs, parametrized by $G / H \times G / H$ (again modulo a finite set), and commutes with $g_{L} H g_{L}^{-1} \times g_{R} H g_{R}^{-1}$ if we choose $E_{L}=g_{L} X g_{L}^{-1}$ (and similarly $E_{R}$ ).

We choose this $K_{2}$ to have a pole at $1 / c$, since

$$
\frac{1}{1-c \theta}(I+c \theta E)=P_{-}+\frac{\frac{N-2 M}{2 N}+\frac{\theta}{i \pi}}{\frac{N-2 M}{2 N}-\frac{\theta}{i \pi}} P_{+}, \quad P_{ \pm}=\frac{1}{2}(I \pm E),
$$

projects at this value onto the restriction to the $S U(M)$ subspace. If the bulk particle has mass $m_{1}$, this gives a boundary bound state (BBS) of mass $m_{1} \sin \left(\frac{2 M}{N}\right)$. This is the starting point of a bootstrap programme in which we can go on to construct the scattering of all the bulk particles (of which there are $N-1$, with $m_{a}=m \sin \frac{a \pi}{N}$ ) off the boundary ground state, and to seek higher BBSs. Finally, of course, we can also scatter the bulk particles off the BBSs themselves. In this context our initial ansatz of a maximum of one pole seems rather natural - it is the scattering of the higher bulk and boundary states which will have more poles. 


\section{Twisted Yangian non-local charges}

The connection between sections two and three is provided by examining the non-local charges which survive on the half-line with these boundary conditions.

In the bulk model, the $G \times G$ symmetry sits inside a larger $Y(\mathbf{g}) \times Y(\mathrm{~g})$ symmetry, where $Y(\mathbf{g})$ is the Yangian algebra. This is generated by charges

$$
\begin{aligned}
Q^{(0) a} & =\int j_{0}^{a} d x \\
Q^{(1) a} & =\int j_{1}^{a} d x-\frac{1}{2} f_{b c}^{a} \int j_{0}^{b}(x) \int^{x} j_{0}^{c}(y) d y d x
\end{aligned}
$$

using $j^{L}$ and $j^{R}$ respectively, decomposed into $j=j^{a} t_{a}$ where the $t^{a}$ are generators of $\mathbf{g}$ with $\left[t_{a}, t_{b}\right]=f_{a b}{ }^{c} t_{c}$. The integrals are over all space, $(-\infty, \infty)$ for the bulk model. But on the half-line $(-\infty, 0]$, these charges are no longer generally conserved. However, there are two important sets of charges which do remain (classically) conserved. Writing $\mathbf{h}$-indices as $i, j, k, .$. and $\mathbf{k}$-indices as $p, q, r, \ldots$, and noting that the only non-zero structure constants are $f^{i}{ }_{j k}$ and $f_{p q}^{i}$ (and cycles thereof), these are

$$
\begin{aligned}
& Q^{(0) i} \\
& \text { and } \quad \widetilde{Q}^{(1) p} \equiv Q^{(1) p}+\frac{1}{2} f_{q i}^{p} Q^{(0) i} Q^{(0) q}
\end{aligned}
$$

as described in [8. charges. After quantization, this second set of charges may be written as

$$
\widetilde{Q}^{(1) p}=Q^{(1) p}+\frac{1}{4}\left[C_{2}^{\mathbf{h}}, Q^{(0) p}\right]
$$

where $\left(\hbar=1\right.$ and) $C_{2}^{\mathbf{h}} \equiv \gamma_{i j} Q^{(0) i} Q^{(0) j}$ is the quadratic Casimir operator of $\mathbf{g}$ restricted to $\mathbf{h}$, with $\gamma_{i j}=f_{i a}{ }^{b} f_{j b}{ }^{a}$. Conservation of these charges can then be used to determine the boundary $S$-matrices, up to an overall scalar factor. The procedure is that, first, conservation of the $Q^{(0) i}$ requires that $K$ act trivially on irreps of $H$ (hence the decomposition of $\left(\overline{3}_{-}-8^{\prime}\right)$ into projectors $P_{ \pm}$). Second, conservation of the $\widetilde{Q}^{(1) p}$ determines the relative values of the coefficients of these projectors in terms of the values of $C_{2}^{\mathbf{h}}$. Details may be found

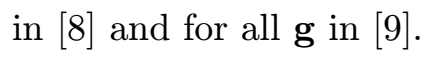

In the same way that $Y(\mathbf{g})$ may be thought of as a deformation of a polynomial algebra $\mathbf{g}[z]$ (with $Q_{1}^{a}=z Q_{0}^{a}$ ), the algebra generated by the $Q^{(0) i}$ and $\widetilde{Q}^{(1) p}$ may be viewed as a deformation of the subalgebra of ('twisted') polynomials in $\mathbf{g}[z]$ invariant under the combined action of $\sigma$ and $z \mapsto-z$. For this reason it is known as the 'twisted Yangian', and we write it $Y(\mathbf{g}, \mathbf{h})$. It is well-known and -studied in the literature (see for example (6ij), although not in this general form. 


\section{Final remarks}

The structures we have described form the foundation for a full boundary bootstrap programme, to discover the boundary spectrum and its interactions with the bulk. Whilst this will be very tough to carry through, some progress has been made $\left[{ }_{-}^{1} \overline{1} \overline{0}\right]$. There are many other directions in which progress might be made - bulk $G / H$ sigma models, models with Wess-Zumino terms and models based on supergroups come to mind - but one of the most intriguing is the apparent unification of various exact $S$-matrix phenomena for exceptional

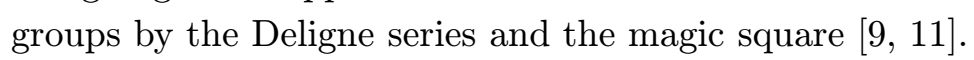

\section{Acknowledgments}

NJM would like to thank the IFT, Sao Paulo for its hospitality, and the Royal Society for a conference/visit grant.

\section{References}

[1] N. MacKay and B. Short, Boundary scattering, symmetric spaces and the principal chiral model on the half-line, hep-th/0104212

[2] J. M. Evans, M. Hassan, N. J. MacKay and A. Mountain, Local conserved charges in principal chiral models, Nucl. Phys. B561 (1999) 385, hep-th/9902008

[3] J. M. Evans and A. J. Mountain, Commuting charges and symmetric spaces, Phys. Lett. B483 (2000) 290, hep-th/0003264

[4] I. V. Cherednik, Factorizing particles on a half-line and root systems, Theor. Math. Phys. 61 (1983) 977

[5] H. Eichenherr, More about nonlinear sigma models on symmetric spaces, Nucl. Phys. B164 (1980), 528, err.ibid. B282 (1987), 745, and

J.-P. Antoine and B. Piette, Classical nonlinear sigma models on Grassmann manifolds of compact or noncompact type, J. Math. Phys. 28 (1987), 2753

[6] A. Molev, M. Nazarov and G. Olshanskii, Yangians and classical Lie algebras, Russ. Math. Surveys 51 (1996) 205

[7] N. MacKay, Rational R-matrices in irreducible representations, J. Phys. A24 (1991) 4017

[8] G. Delius, N. MacKay and B. Short, Boundary remnant of Yangian symmetry and the structure of rational reflection matrices, Phys. Lett. B522 (2001) 335; hep-th/0109115

[9] N. MacKay, Rational K-matrices and representations of twisted Yangians, J. Phys. A35 (2002) 7865; math.QA/0205155

[10] B. Short, Boundary scattering in the $S U(N)$ principal chiral model on the half-line with conjugating boundary conditions, hep-th/0204072, and Ph.D. thesis (in preparation).

[11] B. Westbury, preprint, 2002

P. Dorey, A. Pocklington, R. Tateo, Integrable aspects of the scaling Q-state Potts model: 1. Bound states and bootstrap closure, hep-th/0208111 